\title{
Off-equilibrium dynamics of the frustrated Ising lattice gas
}

\author{
Daniel A. Stariolo ${ }^{1,2, *}$ and Jeferson J. Arenzon ${ }^{2, \dagger}$ \\ ${ }^{1}$ Departamento de Física, Universidade Federal de Viçosa, 36570-000 Viçosa MG, Brazil \\ ${ }^{2}$ Instituto de Física, Universidade Federal do Rio Grande do Sul, Caixa Postal 15051, 91501-970 Porto Alegre RS, Brazil
}

(Received 24 November 1998)

\begin{abstract}
We study by means of Monte Carlo simulations the off-equilibrium properties of a model glass, the frustrated Ising lattice gas in three dimensions. We have computed typical two times quantities, such as densitydensity autocorrelations and the autocorrelation of internal degrees of freedom. We find an aging scenario particularly interesting in the case of the density autocorrelations in real space that is very reminiscent of spin glass phenomenology. While this model captures the essential features of structural glass dynamics, its analogy with spin glasses may make possible its complete description using the tools developed in spin glass theory. [S1063-651X(99)51505-5]
\end{abstract}

PACS number(s): 64.70.Pf, 75.10.Nr, 05.50.+q

Much effort is currently being devoted to reaching a reasonable theoretical understanding of structural glass physics. While the huge amount of experimental work available shows an extremely rich phenomenology, a successful description in terms of microscopic models is still lacking. The theoretical models available are mainly phenomenological, basically focusing the non-Arrhenius relaxation on the socalled fragile glasses [1]. Among the most successful ones are the free volume model [2] and the entropy model [3], in which the relevant variables for the description of a glass transition from a supercooled liquid phase are, respectively, volume and entropy. While the latter model predicts the existence of a thermodynamic second order transition at a well defined temperature, it is extremely difficult to obtain evidence of it since it is, in practice, experimentally inaccessible. Another successful approach has been the mode coupling theory of Götze and Sjögren [4]. This dynamical approach is qualitatively correct in predicting the behavior of time correlations and responses in supercooled liquids. Recently it was shown to correspond to the high temperature limit of a dynamical theory of spin glasses [5]. This raised the interesting possibility of describing the structural glass physics by exploiting the analogy with some spin glasses in which the transition is discontinuous as originally suggested by Kirkpatrick et al. [6]. Moreover, some finite dimensional models have been proposed whose mean field limit would provide such a transition [7]. Nevertheless, although spin and structural glasses have some basic features in common, such as the characteristic slow dynamics, an essential difference is the absence (presence) of quenched disorder in structural glasses (spin glasses). Unlike in spin glasses, the nature of the glass transition may be purely dynamical in origin, without an underlying thermodynamic transition. The existence of a growing correlation length, for example, has not been considered up to very recently in structural glass models [8]. The absence of a simple microscopic model makes these questions very difficult to answer and much of the present knowledge comes from computer simulations of LennardJones systems [9] or from simple kinetically constrained

\footnotetext{
*Electronic address: stariolo@mail.ufv.br

${ }^{\dagger}$ Electronic address: arenzon@if.ufrgs.br
}

models [10-13]. These, in particular, have dynamically selfinduced frustration effects obtained by restricting the possible Monte Carlo movements and reproduce reasonably well the glassy phenomenology. However, although having the advantage of being lattice models, it is not obvious how to relate the ad hoc kinetic rules to the underlying physics.

A possible candidate to fill in this gap is the frustrated Ising lattice gas model (FILG) [14], a Hamiltonian lattice gas in which the presence of internal degrees of freedom subjected to quenched disorder mimics the geometric frustration that slows down the motion of the molecules as the system is cooled or compressed. The introduction of internal degrees of freedom is responsible for the slowing down of the diffusional dynamics of the particles and the necessity of considering them in order to describe the essence of glass physics has recently been addressed by Tanaka [15]. The thermodynamic properties of the FILG in three dimensions (3D) as well as its equilibrium dynamics have been studied by Nicodemi and Coniglio [16]. The model shows many glass properties, including dependence on the cooling (or compression) rate, stretched exponential behavior in correlation functions, a dynamical singularity in which the diffusion constant goes to zero, the breakdown of the Stokes-Einstein relation along with anomalous diffusion at intermediate times. As in many glasses, no singularities in the linear susceptibilities are observed; in particular, the compressibility is continuous everywhere. Scarpetta et al. [17] studied an equivalent, nonlocal version of the FILG [the site frustrated percolation (SFP)] in 2D, finding a behavior similar to the 3D FILG, the main difference being the (Arrhenius) dynamical singularity occurring at zero temperature. This model also seems to relate the glass transition to a percolation-type transition as evidenced by the onset of several precursor phenomena [17].

Nonequilibrium phenomena, aging being one example, are widespread in a great variety of systems, including polymers, granular materials, and ferromagnetic coarsening [19$21]$, and appears in the relaxation dynamics of both spin and structural glasses, both theoretically and experimentally. In this Rapid Communication we report results for the offequilibrium dynamics of the FILG in three dimensions, showing a characteristic aging dynamics present in some two times quantities and some possible scaling scenarios. Of particular interest are the results for the density autocorrelations, 
which suggest the definition of a nonlinear compressibility which may contribute information on a possible thermodynamic transition analog to the magnetic transition in spin glasses.

The FILG is defined by the Hamiltonian

$$
H=-J \sum_{\langle i j\rangle}\left(\varepsilon_{i j} \sigma_{i} \sigma_{j}-1\right) n_{i} n_{j}-\mu \sum_{i} n_{i}
$$

There are two kinds of dynamical variables: the site occupation $n_{i}=0,1 \quad(i=1, \ldots, N)$ and the particles internal degrees of freedom, $\sigma_{i}= \pm 1$. The usually complex spatial structure of the molecules of glass-forming liquids, which can assume several spatial orientations, is in part responsible for the geometric constraints on their mobility. Here we take the simplest case of two possible orientations, and the steric effects imposed on a particle by its neighbors are felt as restrictions on its orientation due to the quenched random variables $\varepsilon_{i j}$ $= \pm 1$. The key role of the first term of the Hamiltonian is that when $J \rightarrow \infty$ (recovering the SFP) no frustrated link can be fully occupied, implying that any frustrated loop in the lattice will have a hole and then $\rho<1$ preventing the system from reaching the close packed configuration. Finally, $\mu$ represents a chemical potential ruling the system density (at fixed volume) and, by taking $\mu \rightarrow \infty$ we recover the EdwardsAnderson spin glass model. It should also be mentioned that, after including gravity, the same model successfully describes granular materials under vibration [22,23], another class of systems where geometric frustration rules its behavior.

By increasing $\mu$ the model presents two characteristic points $[16,17]$. For $\mu \leqslant 0.75$ (low density) it shows liquidlike behavior, time correlation functions decay exponentially, equilibration is quickly achieved, and the particles' mean squared displacement grows linearly with time; a simple diffusion scenario. At $\mu \approx 0.75$ there is a percolation transition (the corresponding density being $\rho \approx 0.38$ ). Dynamically, it manifests itself in the onset of two different relaxation regimes in the correlation functions, a fast exponential relaxation at short times and a slow relaxation at longer times characterized by stretched exponentials. The diffusion is still linear for long times but the diffusion coefficient becomes smaller as the density grows. Also, for a fixed $\mu$, the equilibrium density depends on the cooling rate. The dynamics becomes slower as the chemical potential grows (or equivalently, the temperature is lowered) and a second transition is reached for $\mu \geqslant 6$. This is a spin glass transition associated with the freezing in of the internal degrees of freedom. Interestingly, at this point a dynamical singularity is also present, manifested by the vanishing of the diffusion constant. Besides the qualitative analogies with the physical processes typical of glass-forming liquids, there are a few points of more fundamental character. The presence of two characteristic temperatures (or chemical potentials) separating different dynamical regimes is common with a class of mean field theories of spin glasses and also with what is observed in real glass formers. The percolation transition corresponds to the dynamical transition in mean field $p$-spin or Potts glasses or with the glass transition in the mode coupling theory. In the FILG the dynamical signature of the transition is a consequence of the appearance of percolating clusters, a

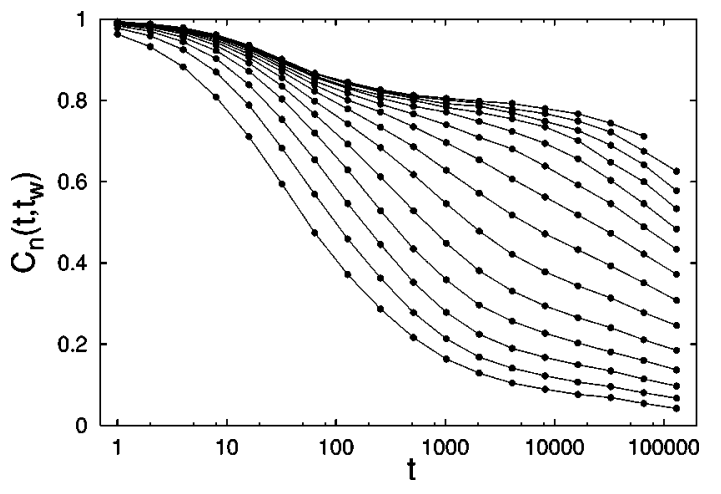

FIG. 1. Density autocorrelations after a quench to $\mu=10$ at $T$ $=1$ and $J=10$ for $L=20$. The waiting times range from $2^{5}$ (bottom) to $2^{17}$ (top) and the averages are over 50 samples.

geometrical feature that may be responsible for the slowing down of the dynamics in structural glasses. The second transition corresponds to the ideal glass transition at which the relaxation times diverge and the diffusion constant goes to zero. In the FILG the structural manifestation of this transition is the presence of a frozen percolating cluster. The density of the system approaches a critical value $\rho_{c} \approx 0.7$, attainable only by an infinitely slow cooling. These facts make the FILG an important model for studying different aspects of the glass transition.

Different protocols can be envisaged for studying, for example, correlations and response functions. We have prepared the model in a nonequilibrium state, setting the parameters as in [16] with an initial density lower than the critical one. Then the system is quenched to a supercritical chemical potential and dynamic correlations are recorded. We have used a Monte Carlo dynamics that alternates flipping of the internal degrees of freedom, creation and destruction of particles in a plane surface (which mimics a compression experiment) as in [11] and particle diffusion. The creationdestruction of particles is fast enough to destroy all particles at long times if it is allowed in the bulk.

Consider the connected two-point correlation

$$
c\left(t, t_{w}\right)=\frac{1}{N} \sum_{i} n_{i}\left(t+t_{w}\right) n_{i}\left(t_{w}\right)-\rho\left(t+t_{w}\right) \rho\left(t_{w}\right),
$$

where the global density at time $t$ is given by $\rho(t)$ $=N^{-1} \sum_{i} n_{i}(t)$. We now define the density autocorrelations as $C_{n}\left(t, t_{w}\right)=c\left(t, t_{w}\right) / c\left(0, t_{w}\right)$. In Fig. 1 the behavior of $C_{n}\left(t, t_{w}\right)$ is shown as a function of $t$ in a semilog plot after a quench in chemical potential to a value $\mu=10$, for waiting times between $2^{5}$ and $2^{17}$. A typical aging scenario is present, signaling the slowing down of the dynamics as the waiting time grows. For the longest waiting times the correlation presents a rather fast relaxation to a plateau in which the system is in quasiequilibrium: the dynamics is stationary and the fluctuation-dissipation relations hold. The plateau separates two time scales typical of glassy systems: a $\beta$ (fast) relaxation for small time and an $\alpha$ (slow) relaxation at longer times, corresponding, respectively, to the fast movements of the particle inside the dynamical cages and the large scale, cooperative process that takes much more time in order to rearrange the cages. Moreover, in this very long time regime $\left(t \gg t_{w}\right)$, the system falls out of equilibrium, the correlations decay to zero asymptotically, and time translational invari- 


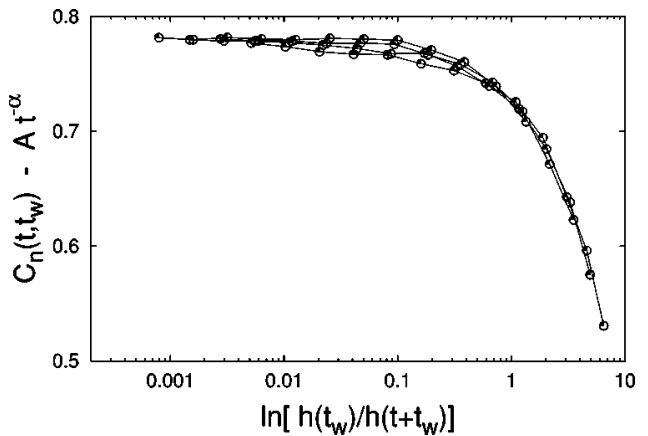

FIG. 2. Scaling plot of the five upper curves of Fig. 1. The initial five points ( $\beta$ decay) were discarded and the parameters are $A=0.59, \alpha=0.46$, and $\mu=0.9$. See text for details.

ance (TTI) no longer holds with the corresponding violation of the fluctuation-dissipation theorem (FDT).

Since the $C_{n}\left(t, t_{w}\right)$ curves have a complex behavior, it is possible to scale the curves only after a careful analysis of the different time scales present $[24,25]$. It is clear that only for the largest waiting times can different relaxation scales be observed. We restrict our analysis to the five upper curves $\left(t_{w}=2^{13}, \ldots, 2^{17}\right)$. In these curves, a fast initial decay is observed up to times $t \sim 100 \mathrm{MCS}$, corresponding to the already mentioned $\beta$ decay, which we will not analyze in any more detail. Then a plateau characteristic of quasiequilibrium dynamics develops. Its asymptotic value as $t_{w} \rightarrow \infty$ defines the ergodicity breaking (or Edwards-Anderson) parameter. For a fixed, large $t_{w}$ and $t \gg t_{w}$, the system begins to fall out of equilibrium and the correlations decay to zero. But only the very early epochs of this decay can be observed within the times of our simulations, although we can observe the crossover between equilibrium and nonequilibrium dynamics. Consequently, in order to obtain a good scaling for this last regime we have subtracted from $C_{n}\left(t, t_{w}\right)$ a stationary contribution of the form

$$
C_{n}\left(t, t_{w}\right)=C_{\infty}+A t^{-\alpha}, \quad t_{w} \text { fixed, } t \ll t_{w},
$$

with $C_{\infty}, A$ and $\alpha$ as fit parameters. For the nonstationary regime we assumed a time dependence of the form $h\left(t_{w}\right) / h\left(t+t_{w}\right)$ with $h(x)$ given by [24] $h(x)=\exp [(1$ $\left.-\mu)^{-1}(x / \tau)^{1-\mu}\right]$, with $\mu<1$ and $\tau$ a microscopic time scale. Note that this form is quite general as one recovers the cases of a simple $t / t_{w}$ dependence (full aging) when $\mu=1$ and stationary dynamics when $\mu=0$. The final scaling is shown in Fig. 2. The scaling obtained is much better than assuming only full aging or activated dynamics, even after including in these cases the contribution from the stationary decay.

We have also measured, as shown in Fig. 3, the autocorrelations of the internal degrees of freedom,

$$
C_{s}\left(t, t_{w}\right)=\frac{1}{N} \sum_{i} s_{i}\left(t+t_{w}\right) n_{i}\left(t+t_{w}\right) s_{i}\left(t_{w}\right) n_{i}\left(t_{w}\right) .
$$

The internal degrees of freedom correspond to a diluted Edwards-Anderson (EA) spin glass and the curves should be compared to the ones of the 3D EA model [26]. From our knowledge of the 3D EA model one may expect the scaling of the autocorrelation in the aging regime be of the form $t^{-\alpha} \widetilde{C}\left(t / t_{w}\right)$. Nevertheless we verified that an activated dy-

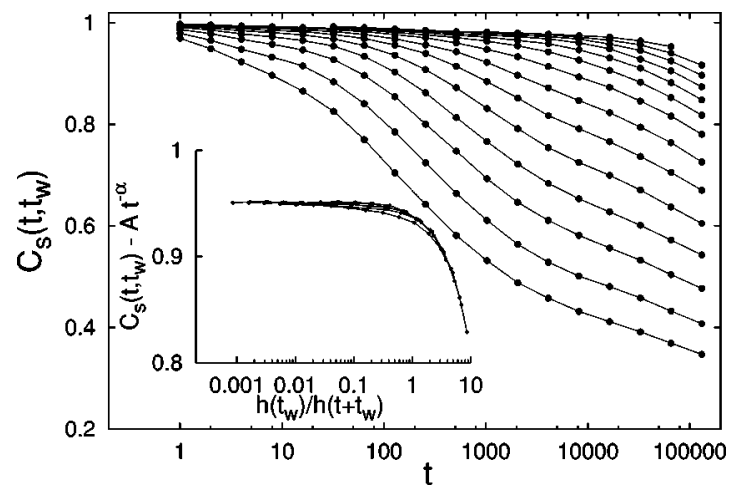

FIG. 3. Autocorrelation of the internal degrees of freedom after a quench to $\mu=10$ with $T=1$ and $J=10$ for $L=20$. Waiting times from $2^{5}$ to $2^{17}$ (top to bottom) and average over 50 samples. Inset: scaling with $\tau_{0}=0.5, \mu=0.9, \alpha=0.091$, and $A=0.057$ (see text).

namics scaling of the form $C_{s}\left(t, t_{w}\right) \propto \widetilde{C}\left[\log (t) / \log \left(t_{w}\right)\right]$ works better. Moreover, doing an analysis similar to the one for $C_{n}\left(t, t_{w}\right)$ the scaling can be slightly improved (see Fig. 3, inset).

This Hamiltonian lattice model presents the essentials of structural glass phenomenology. In particular, the results for the density autocorrelation are very promising, suggesting that it may be possible to apply spin glass ideas and techniques for an analytical investigation of the model [18]. The similarity between the density autocorrelation and the spin glass overlap function suggests the introduction of a nonlinear compressibility in analogy with the spin glass susceptibility, which may be a good quantity for studying the possibility of an underlying phase transition with a growing correlation length in the model. Another issue that must be studied is the precise form of the violation of FDT through the so-called fluctuation dissipation ratio [27]. Also, other protocols may be implemented in order to probe the off equilibrium dynamics of the system. Work in progress, keeping fixed the global density and performing a quench in temperature, indicates that the phenomenology is similar to what we have presented here. Moreover, the evaluation of the root mean square deviation of the particles and the incoherent scattering function (which is related to the Fourier transform of the density correlations) also show evidence of slow dynamics and aging. The results of these investigations will be presented in a future publication. We are working also in the 2D version of the model: here, some differences are expected with respect to $3 \mathrm{D}$ because the dynamical singularity only occurs for $T=0 \quad(\mu \rightarrow \infty)$ and the relaxation time diverges with an Arrhenius law. Still, other issues can also be explored. For instance, to what extent, if any, the scenario for the 3D model presented here changes as one goes to infinite range connections. In the mean field version, where both first and second order transitions show up, it might be possible that different aging regimes are present [18], and it would be interesting to know if the FILG is a finite-dimensional version of a model whose mean field limit has a discontinuous transition.

This work was partially supported by the Brazilian agencies CNPq and FAPEMIG. We acknowledge M. Sellitto for a careful reading of the manuscript and J.A.C. Gallas for providing us with time on his alpha station. 
[1] C.A. Angell, Science 267, 1924 (1995); R. Böhmer, K.L. Ngai, C.A. Angell, and D.J. Plazek, J. Chem. Phys. 99, 4201 (1993).

[2] M.H. Cohen and D. Turnbull, J. Chem. Phys. 31, 1164 (1959); D. Turnbull and M.H. Cohen, ibid. 34, 120 (1961); M.H. Cohen and G.S. Grest, Phys. Rev. B 20, 1077 (1979); G.S. Grest and M.H. Cohen, ibid. 21, 4113 (1980).

[3] J.H. Gibbs and E.A. di Marzio, J. Chem. Phys. 28, 373 (1958); G. Adam and J.H. Gibbs, ibid. 43, 139 (1965).

[4] W. Götze and L. Sjögren, Rep. Prog. Phys. 55, 241 (1992); W. Götze, in Liquids, Freezing and the Glass Transition, Proceedings of the Les Houches Summer School of Theoretical Physics, 1989, edited by J.P. Hansen, D. Levesque, and J. ZinnJustin (North Holland, Amsterdam, 1991).

[5] J.P. Bouchaud, L.F. Cugliandolo, J. Kurchan, and M. Mézard, Physica A 226, 243 (1996).

[6] T.R. Kirkpatrick and D. Thirumalai, Phys. Rev. Lett. 58, 2091 (1987); T.R. Kirkpatrick and D. Thirumalai, Phys. Rev. B 36, 5388 (1987); T.R. Kirkpatrick, D. Thirumalai, and P.G. Wolynes, Phys. Rev. A 40, 1045 (1989).

[7] H. Rieger, Physica A 184, 279 (1992); J. Kisker, H. Rieger, and H. Schreckenberg, J. Phys. A 27, L853 (1994); D. Alvarez, S. Franz, and F. Ritort, Phys. Rev. B 54, 9756 (1996); S. Franz and G. Parisi, e-print cond-mat/9805088.

[8] D. Lancaster and G. Parisi, J. Phys. A 30, 5911 (1997); G. Parisi, e-print cond-mat/9801034.

[9] W. Kob and J.L. Barrat, Phys. Rev. Lett. 78, 4581 (1997); G. Parisi, ibid. 79, 3660 (1997).

[10] G.H. Fredrickson and H.C. Andersen, Phys. Rev. Lett. 53, 1244 (1984); J. Chem. Phys. 83, 5822 (1985); W. Kob and H.C. Andersen, Phys. Rev. E 48, 4364 (1993).

[11] J. Kurchan, L. Peliti, and M. Sellitto, Europhys. Lett. 39, 365 (1997); L. Peliti and M. Sellitto, in Disorder and Chaos, edited by A. Vulpiani (Editions de Physique, Rome, 1998); M. Sellitto, Eur. Phys. J. B 4, 135 (1998).

[12] E. Follana and F. Ritort, Phys. Rev. E 54, 930 (1996).
[13] I.S. Graham, L. Piché, and M. Grant, Phys. Rev. E 55, 2132 (1997).

[14] A. Coniglio, Nuovo Cimento D 16, 1027 (1994); A. Coniglio, Philos. Mag. B 77, 213 (1998).

[15] H. Tanaka, J. Phys. C 10, L207 (1998).

[16] M. Nicodemi and A. Coniglio, J. Phys. A 30, L187 (1997); Phys. Rev. E 57, R39 (1998).

[17] S. Scarpetta, A. de Candia, and A. Coniglio, Phys. Rev. E 55, 4943 (1997); G. Franzese and A. Coniglio, ibid. (to be published).

[18] J. J. Arenzon, M. Nicodemi, and M. Sellitto, J. Phys. I 6, 1143 (1996); M. Sellitto, M. Nicodemi, and J.J. Arenzon, ibid. 7, 45 (1997).

[19] L. C. E. Struik, Physical Aging in Amorphous Polymers and Other Materials (Elsevier, Amsterdan, 1978).

[20] J. P. Bouchaud, L. F. Cugliandolo, J. Kurchan, and M. Mézard, in Spin Glasses and Random Fields, edited by P. Young (World Scientific, Singapore, 1998).

[21] A.J. Bray, Adv. Phys. 43, 3 (1994).

[22] A. Coniglio and H. Herrmann, Physica A 220, 1 (1996); M. Nicodemi, A. Coniglio, and H. Herrmann, J. Phys. A 30, L379 (1997); Phys. Rev. E 55, 3962 (1997).

[23] J. J. Arenzon, J. Phys. A 32, L107 (1999).

[24] E. Vincent, J. Hammann, M. Ocio, J.-P. Bouchaud, and L. F. Cugliandolo, Proceedings of the Sitges Conference on Glassy Dynamics, edited by E. Rubi (Springer, Berlin, 1996).

[25] M. Nicodemi and A. Coniglio, Phys. Rev. Lett. 82, 916 (1999); U. Müssel and H. Rieger, ibid. 81, 930 (1998); W. Kob and J.-L. Barrat, ibid. 81, 931 (1998).

[26] H. Rieger, J. Phys. A 26, L615 (1993).

[27] J.-O. Andersson, J. Mattson, and P. Svedlindh, Phys. Rev. B 46, 8297 (1992); L. Cugliandolo and J. Kurchan, Phys. Rev. Lett. 71, 173 (1993); S. Franz, M. Mèzard, G. Parisi, and L. Peliti, ibid. 81, 1758 (1998); L. Cugliandolo, J. Kurchan, and L. Peliti, Phys. Rev. E 55, 3898 (1997). 